\title{
PAX2 regulates NRP-1 expression in renal tubular epithelial cellsto promote epithelial-to-mesenchymal transition and renal fibrosis
}

\author{
Ling Hou, Yue Du, Chengguang Zhao, Xiuli Wang, Yubin Wu*
}

Department of pediatrics, Shengjing Hospital of China Medical University, Shenyang,

\begin{abstract}
To observe the expression of neuropilin-1 (NRP-1) induced by paired-box gene 2 (PAX2) during the process of epithelial-to-mesenchymal transition (EMT) and renal fibrosis in unilateral ureteral obstruction (UUO) model in rats, and explore the mechanism of EMT induced by PAX2. Methods: The recombinant lentivirus expression vector for PAX2 was constructed and transfected into rat normal renal tubular epithelial cell line (NRK52E). The experimental cells were divided into three groups: transfection group, empty vector group, and normal group. E-cadherin and oup, empty vector group, and normal and real-time PCR. Expression of NRP-1 was detected by western blot, real-time PCR, and immunofluorescence. Sixty male Wistar rats were randomly divided into two groups: the sham-operation group $(\mathrm{n}=30)$ underwent left ureteral dissection, the UUO group $(\mathrm{n}=30)$ underwent left ureteral ligation. Post-operation on days 3,7 , 14, 21 and 28, 6 rats from each of the groups were sacrificed and the obstructed kidneys were dissected out. The histopathological changes were observed by hematoxylin-eosin and Masson staining. E-cadherin and dhSMA were detected by western blot and immunohistochemistry. Expression of NRP-1 and PAX2 were determined by western blot, immunohistochemistry, and real-time PCR. Results: Expression of NRP-1 mRNA and protein and d by western blot, immunoh $<0.05)$ while E-cadherin protein expression decreased $(\mathrm{P}<0.05)$ in the transfection group as compared to the empty vector group in vitro. In the UUO group, fibrosis was obvious, and there was decreased expression of E-cadherin protein $(\mathrm{P}<0.05)$ and increased expression of 5$)$ and increased expression in (Pious, and $<0.05)$ in comparison to the sham group. Conclusion: NRP-1 maybe mediate PAX2-induced EMT in renal tubular epithelial cells and renal fibrosis.
\end{abstract}

Keywords: renal interstitial fibrosis; ureteral obstruction; epithelial-to-mesenchymal transition; paired-box gene 2; neuropilin-1

\section{Introduction}

Renal interstitial fibrosis (RIF) can cause progressive renal injury, and is a common pathway by which chronic kidney disease progress to end-stage renal disease ${ }^{[1]}$. Renal tubular epithelial-to-mesenchymal transition (EMT) is an important mechanism underlying the development of $\operatorname{RIF}^{[2]}$. Paired-box gene 2 (PAX2) encodes a nuclear transcription factor and is a key gene for embryonic kidney development ${ }^{[3,4]}$. PAX2 is not expressed in mature kidneys, but it is expressed following renal injury ${ }^{[5]}$. Our preliminary study indicated that PAX2 can causes RIF via EMT ${ }^{[6,7]}$, and we subsequently found that PAX2 could induce EMT in normal renal tubular epithelial cells in vitro ${ }^{[8]}$, which indicates that PAX2 is associated with the development of RIF. However, the mechanisms involved in how PAX2 induces EMT are not well understood. By analysis of gene expression profiling of rat cDNA gene chip, we obtained information of mRNA differential expression between the PAX2 overexpression group and the empty vector group. We observed that when PAX2 was overexpressed in the renal tubular epithelial cells, the mRNA expression of neuropilin-1 (NRP-1) was upregulated by 4.06 fold (the data is not published). NRP-1, initially discovered in the developing nerve fiber axons, is a receptor of semaphorin 3A and vascular endothelial growth factor (VEGF)-165 and is mainly expressed in glomerular epithelial cells and collecting ducts ${ }^{[9]}$. Studies have suggested that NRP-1 can induce EMT in tumor cells ${ }^{[10]}$, and its expression is increased in hepatic fibrosis ${ }^{[1,12]}$. Considering the importance of PAX2-induced EMT in renal tubular epithelial cells and its relationship with NRP-1, we selected NRP-1 as a candidate target gene and we hypothesized that PAX2 regulates NRP-1 expression in renal tubular epithelial cells, and that NRP-1 is involved in RIF. In this study, we observed NRP-1 expression in renal tubular epithelial cells with PAX2 overexpression and in the unilateral ureteral obstruction (UUO) model in rats and explored the mechanism of EMT induced by PAX2.

Copyright (C) 2018 Ling Hou et al.

doi: 10.24294/jpd.v2i1.174

EnPress Publisher LLC.This work is licensed under the Creative Commons Attribution-NonCommercial 4.0 International License (CC BY-NC 4.0).

http://creativecommons.org/licenses/ by/4.0/ 


\section{Materials and Methods}

1. Transfection: The rat normal renal tubular epithelial cell line (NRK52E) was purchased from Shanghai Cell Bank of Chinese Academy of Sciences, and the recombinant lentivirus expression vector for PAX2 (pGC-LV-PAX2) was constructed in the laboratory preliminarily. The pGC-LV-PAX2 was transfected into the NRK52E cell line. The experimental cells were divided into three groups: transfection group, empty vector group, and normal group. The cells were cultured in high-glucose DMEM medium containing $10 \%$ fetal bovine serum at $37{ }^{\circ}$ ere cultured in high-glucose DMEM medium containing 10\% fetal bovine serum at 37ividedHyclone), trypsin (Gibco), fetal bovine serum (Hyclone) and penicillin-streptomycin (Gibco).

2. Animal models and grouping: Sixty healthy male Wistar rats $(120-150 \mathrm{~g}, 4-6$ weeks old; provided by the Experimental Animal Center, Shenging Hospital of China Medical University) were randomly assigned to the shamoperation group $(n=30)$ and the UUO group $(n=30)$. At 3, 7, 14, 21 and 28 days after the operation, 6 rats from each group were sacrificed. The UUO rat model was established as follows: The rats were anesthetized by the intraperitoneal injection of $10 \%$ chloral hydrate $(0.03 \mathrm{~mL} / \mathrm{kg})$ and then placed on the operation bench in a supine position. The limbs and head were fixed, and an incision was made in the left abdomen. The left ureter was exposed along the inferior pole of left kidney and dissected, ligated at two sites (up and down) using 4-0\# silk suture and cut off at the mid-point to prevent infection. Finally, the abdomen was closed and sutured layer by layer. In the sham group, the abdomen was opened by the same procedure and then sutured after the left ureter was only dissected without ligation.

3. Histological examination of kidneys

Paraffin-embedded renal sections (4 $\mu$ a) were subject to hematoxylin-eosin (HE) staining to evaluate the morphological changes in kidneys and Masson staining to observe tubulointerstitial lesions.

4. Real-time Quantitative PCR (Real-time qPCR)

Total RNA of rat renal tubular epithelial cells and kidneys were extracted with RNAiso Plus (TAKARA, Japan) and cDNA was synthesized with PrimeScriptTM RT reagent kit (TAKARA) according to manufacturer manufacturerording $t$ ng total RNA and 202L reaction system were used for reverse transcription, and $1 \mu \mathrm{L}$ of the product from reverse transcription was used for Real-time qPCR. PCR was performed with SYBR ${ }^{\circledR P r e m i x}$ Ex TaqTM (Tli RNaseH Plus) (TAKARA) on Applied Biosystems 7500 according to the standard two-step PCR protocol in the operation manual; PCR primers were synthesized by Sangon Biotech (Shanghai) Co., Ltd (Table 1).

Table1. Primers and amplicons used for real-time qPCR.

\begin{tabular}{|c|c|c|c|}
\hline \multicolumn{2}{|c|}{ Primers } & Sequences & Product size(bp) \\
\hline \multirow[t]{2}{*}{ NRP1 } & Forward & 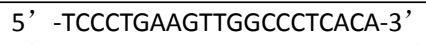 & 145 \\
\hline & Reverse & 5' -AGCACGCTGTAGTTGGCTGAG-3' & \\
\hline \multirow[t]{2}{*}{ PAX2 } & Forward & 5' -ACGAGACTGGCAGCATCAA-3’ & 104 \\
\hline & Reverse & 5' -CGGGTTCTGTCGCTTGTATT-3' & \\
\hline \multirow[t]{2}{*}{ E-cadherin } & Forward & 5’ '-TGCTCCTACTGTTTCTACG-3' & 111 \\
\hline & Reverse & 5' - -СТТСТССАССТСССТСТТ -3' & \\
\hline \multirow[t]{2}{*}{ a -SMA } & Forward & 5' -AGCCAGTCGCCATCAGGAAC-3' & 90 \\
\hline & Reverse & 5' -CCGGAGCCATTGTCACACAC-3' & \\
\hline \multirow[t]{2}{*}{ GAPDH } & Forward & 5' -TTCAACGGCACAGTCAAGG-3’ & 114 \\
\hline & Reverse & 5' -CTCAGCACCAGCATCACC-3, & \\
\hline
\end{tabular}

5. Immunofluorescence staining

Cells were fixed with $4 \%$ paraformaldehyde for $10 \mathrm{~min}$, permeabilized with $0.5 \%$ Triton X-100 for 20 min, blocked with normal goat serum, incubated with primary antibody (NRP-11:100, Abcam ab81321, USA) at 4 at 4 goat serum, incubated with primary antibody (NRP-11t IgG(H+L), Alexa Fluor 594, Invitrogen, USA) at room temperature for $2 \mathrm{~h}$, stained with DAPI, mounted, and observed under fluorescence microscope.

6. Immunohistochemistry

Paraffin-embedded renal sections $(4 \mu \mathrm{a})$ were deparaffinized with xylene, hydrated with gradient alcohol, exposed to citric acid antigen retrieval solution (Fuzhou Maixin Biotech. Co., Ltd.), and then heated for $10 \mathrm{~min}$ in a microwave oven for antigen retrieval; thereafter, $3 \%$ hydrogen peroxide was added to inactivate endogenous peroxidase, and blocked with normal goat non-immune serum. The sections were incubated at $4{ }^{\circ}$ in in a microwave oven for antigen retrieval; theAbcam ab81321, USA; E-cadherin1:50, Abcam ab76055; 055; E-cadheAbcam ab5694; PAX2 1:50, sc-130387, Santa Cruz biotechnology, USA), dripped with biotin-labeled secondary antibodies, followed by SP reagent, and finally DAB.

7. Protein extraction and western blot

The cells were washed with PBS, while renal tissues were sheared into small pieces on ice, followed by addition of a mixture of RIPA lysis buffer and PMSF (100:1), and then homogenized by ultrasound, preserved on ice for 30 min, and thereafter, centrifuged at a low temperature for $30 \mathrm{~min}$. The supernatant was removed, and the protein concentration was measured by BCA assay (Thermo Scientific Pierce BCA Protein Assay). A total protein of $40 \mu$ was used for 10\% SDSPAGE gel electrophoresis, followed by transfer onto a $0.45 \mu \mathrm{w}$ PVDF membrane and then protein transmembrane 
electrophoresis was carried out at $100 \mathrm{~V}$ for $100 \mathrm{~min}$, blocked with $5 \%$ defatted milk powder for $1 \mathrm{~h}$, followed by incubation at $4{ }^{\circ}$, followed by incubation at 4milk powder for 10lecAbcam ab81321, USA; E-cadherin 1:1000, Abcam ab76055; 055; E-cadherAbcam ab5694; PAX21:200, sc-130387, Santa Cruz biotechnology, USA; nology1:200, sc1Beijing DingguoChangsheng Biotechnology Co., Ltd.). The treated membrane was incubated separately with horseradish peroxidase (HRP)-labeled secondary antibodies (ZSGB-BIO) for $2 \mathrm{~h}$, and then detected by electrochemiluminescence (ECL, Pierce ECL Western Blotting kit).

8. Statistical Analysis

SPSS18.0 statistical software was used for statistical analysis. The data is presented as the mean e (HRP)-labeled secondary antibodies (ZSGB-BIO) for s (ZSGB-BIO) VA and LSD-t test. $\mathrm{P}<0.05$ suggested that the difference was statistically significant.

\section{Results}

1. PAX2 increased the transcription and expression of NRP-1 while inducing EMT in renal tubular epithelial cells.

Real-time qPCR and western blot demonstrated that when PAX2 transfected into renal tubular epithelial cells, the mRNA and protein expression of E-cadherin (an epithelial marker) decreased, while those of $\alpha$-SMA (a mesenchymal marker) increased, as compared to the empty vector group (Figure 1, $\mathrm{P}<0.05$; Figure 2, $\mathrm{P}<0.05$ ), which is consistent with the results of our preliminary study showing that PAX2 could induce EMT in renal tubular epithelial cells ${ }^{[6]}$. In addition, the results of western blot and immunofluorescence studies revealed that the expression of NRP-1 protein in PAX2 transfection group was higher than that in the empty vector group (Figure 2, P $<0.05$; Figure 3). Real-time qPCR results demonstrated that the mRNA expression of NRP-1 in PAX2 transfection group increased (Figure $1, \mathrm{P}<0.05$ ), which is consistent with the rat cDNA microarray screening results obtained by our research group.

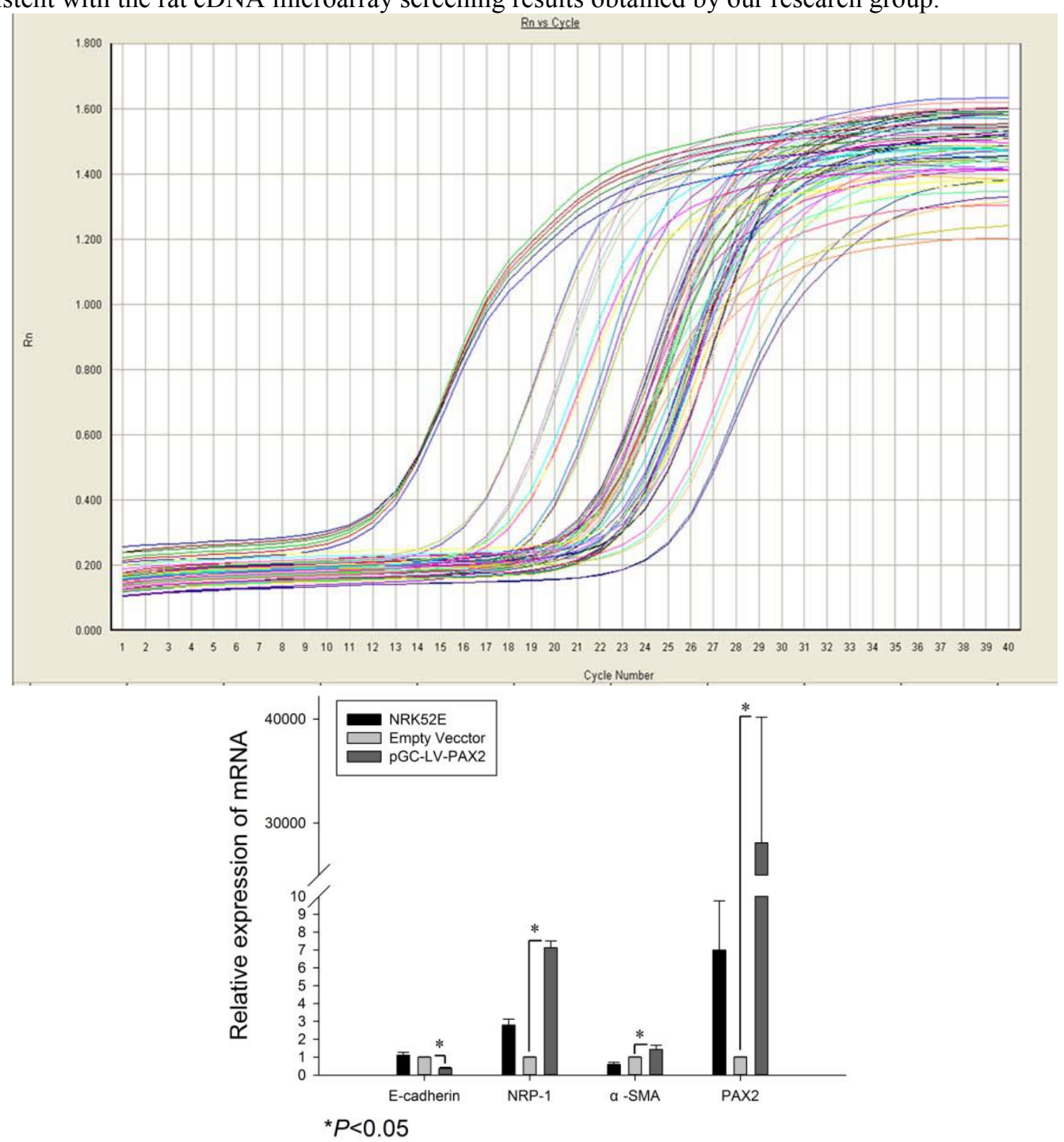

Figure 1. Messenger RNA was isolated from NRK52E cells that were transfected with pGC-LV-PAX2 or empty vector. Data represent mean $\pm \mathrm{SD}(\mathrm{n}=3) . * \mathrm{P}<0.05$ compared to vector control. 


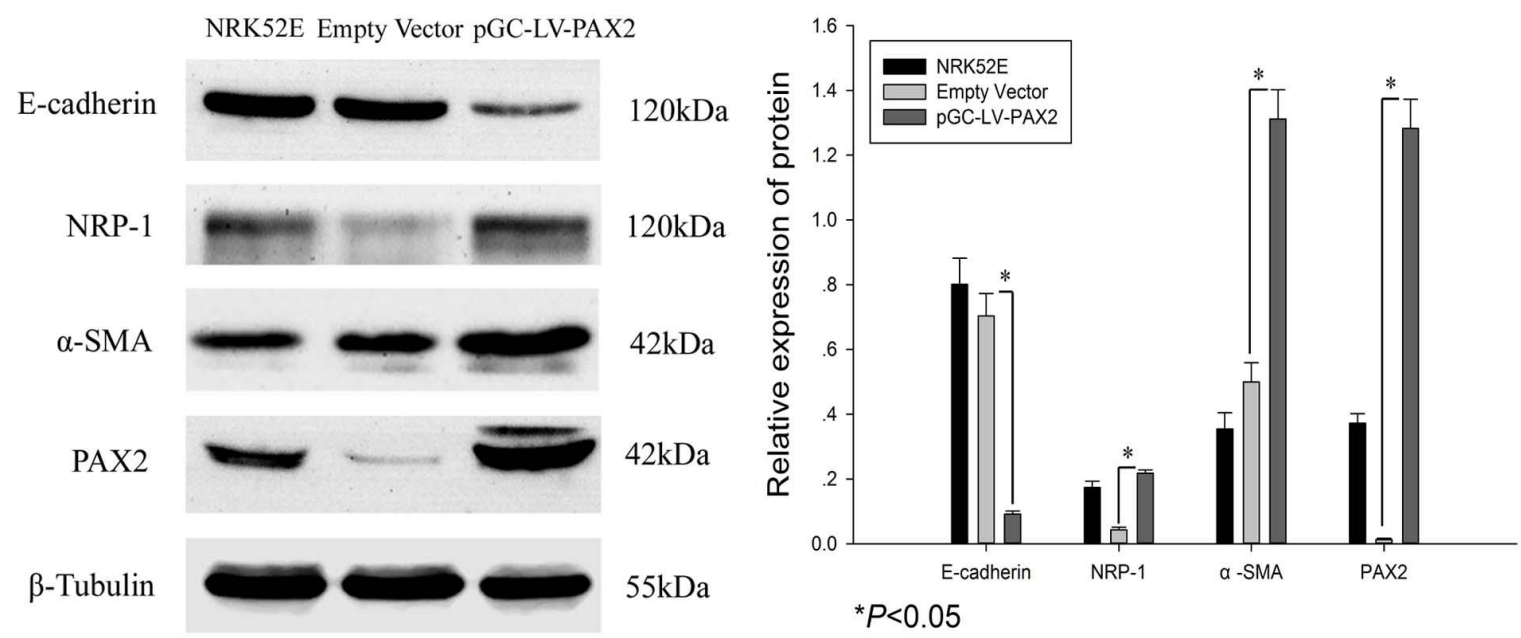

Figure 2. Total cell lysates of NRK52E cells transfected with pGC-LV-PAX2 was prepared and western blot analysis was performed. As a control, empty vector was used. Polyvinylidene fluoride membranes were incubated with antibodies against NRP-1, PAX2, E-cadherin and $\alpha$-SMA. Anti- $\beta$-tubulin was used to determine equal protein loading. Data represent mean \pm e SD $(n=3)$.
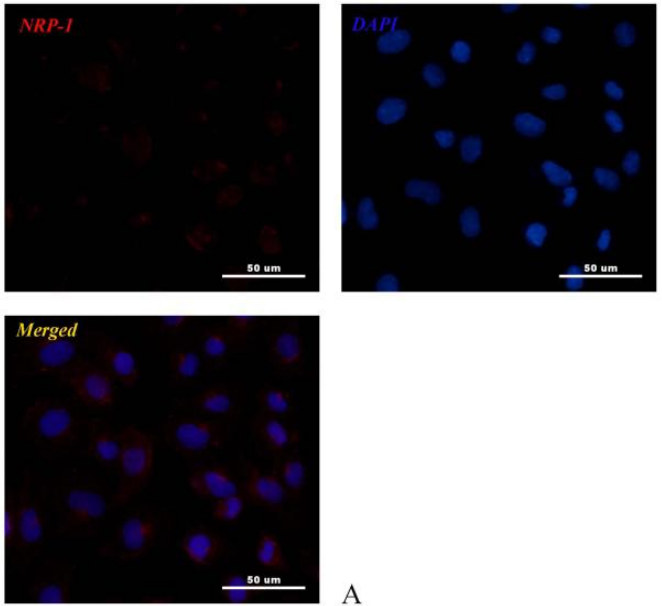
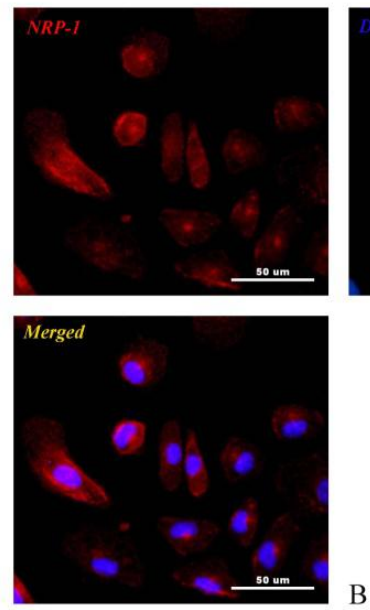

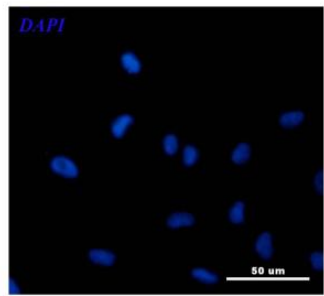

B

Figure 3. NRP-1 expression (red) was analyzed by immunofluorescence analysis of NRK52E cells transfected with pGC-LV-PAX2 or empty vector. Nuclei of NRK52E cells were stained with 4', 6-diamidino-2-phenylindole (DAPI). Scale bars represent 50 witA: empty vector control; B: PAX2 transfection group

\section{Histological changes of rat kidneys in the UUO group}

Hematoxylin-eosin and Masson staining results showed that in the sham group, rat renal glomeruli and tubules had a normal appearance. However, in the UUO group, renal tubular epithelial cells were swollen and vacuolized, renal tubules were atrophic with dilated lumens, and there was diffuse mononuclear phagocyte and lymphocytes infiltrating into the interstitium. The interstitium was widened, collagen fibers were increased, fibrosis exacerbated over the obstruction time, most of the microvascular lumen was compressed, a part of microvascular lumen was disintegrated or dilated, and the lumen structure disappeared (Figure 4 and Figure 5).

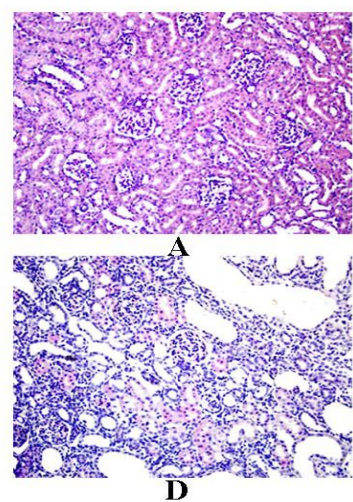

D

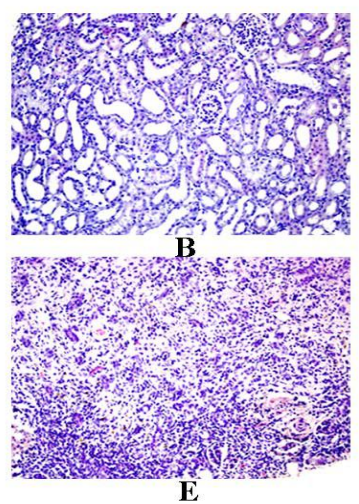

E

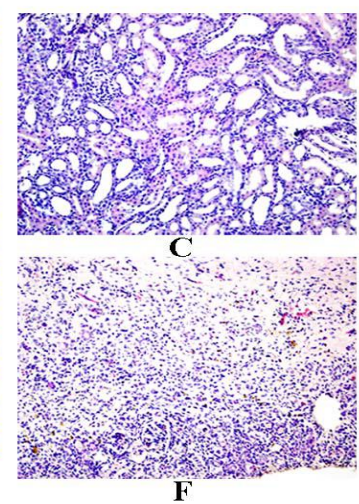

F

Figure 4. H\&E staining of UUO kidneys in various groups (200men A: sham group; B: 3d group; C: 7d group; D; 14d group; E: 21d group; F: 28d group 

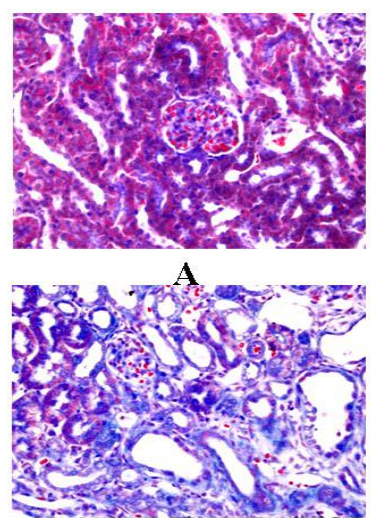

D

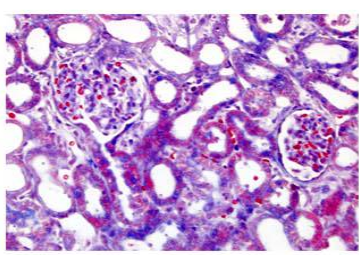

B

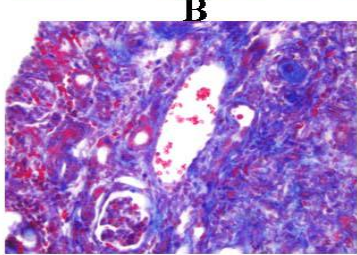

E
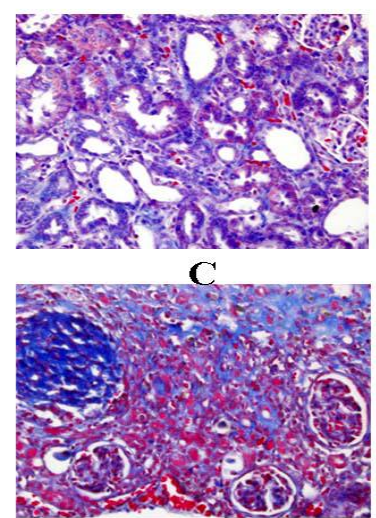

$\mathbf{F}$

Figure 5. Masson staining of UUO kidneys in various groups (400ructA: sham group; B: 3d group; C: 7d group; D; 14d group; E: 21d group; F: 28d group.

3. NRP-1 was involved in renal fibrosis in UUO rats

In order to explore the relationship between NRP-1 and renal fibrosis in vivo, we evaluated the NRP-1 expression in the process of RIF induced by ureteral obstruction. Immunohistochemistry and western blot studies indicated that in the renal tissues of these animals, the expression of E-cadherin protein decreased (Figure 6 and Figure 7, P $<0.05$ ) and that of NRP-1 expressionFigure 8 and Figure $7, \mathrm{P}<0.05$ ) during the study, suggesting that the EMT of renal tubular epithelial cells occurred in the UUO model. As shown in Figure 9, NRP-1 was mainly located in the cytoplasm and membrane of renal tubular epithelial cells, and its expression in fibrotic kidneys increased, which was consistent with the expression of PAX2 (Figure 10). Western blot demonstrated that the expression of NRP-1 protein was markedly enhanced on day 3 of UUO, and there was also a trend of increase consistent with the protein expression of PAX2 (Figure 7, P < 0.05). Real-time qPCR showed that NRP-1 mRNA level in the UUO group was higher than that of the sham group, and its increase was coincident with the increase of PAX2 mRNA level (Figure 11, P $<0.05$ ).
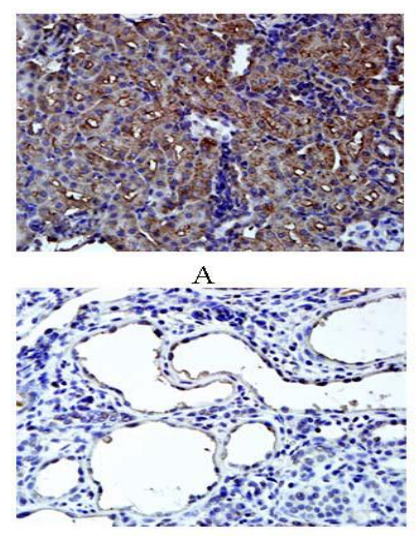

$\mathrm{D}$

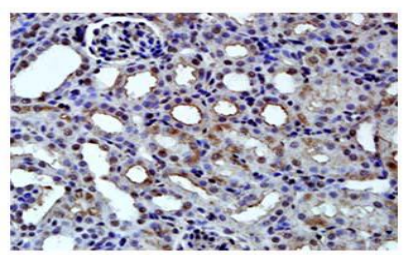

$\mathrm{B}$

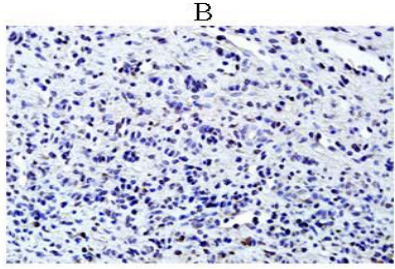

E

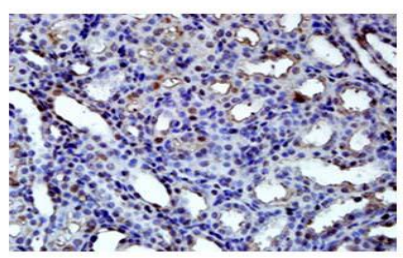

$\mathrm{C}$

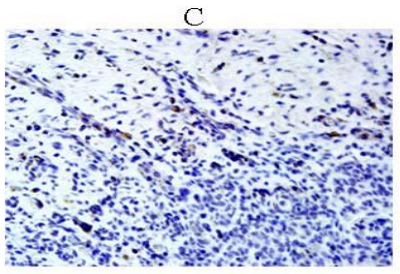

F

Figure 6. Protein expression of E-cadherin in the sham group and the UUO group at various time points detected by immunohistochemistry (400ncr A: sham group; B: 3d group; C: 7d group; D; 14d group; E: 21d group; F: 28d group.
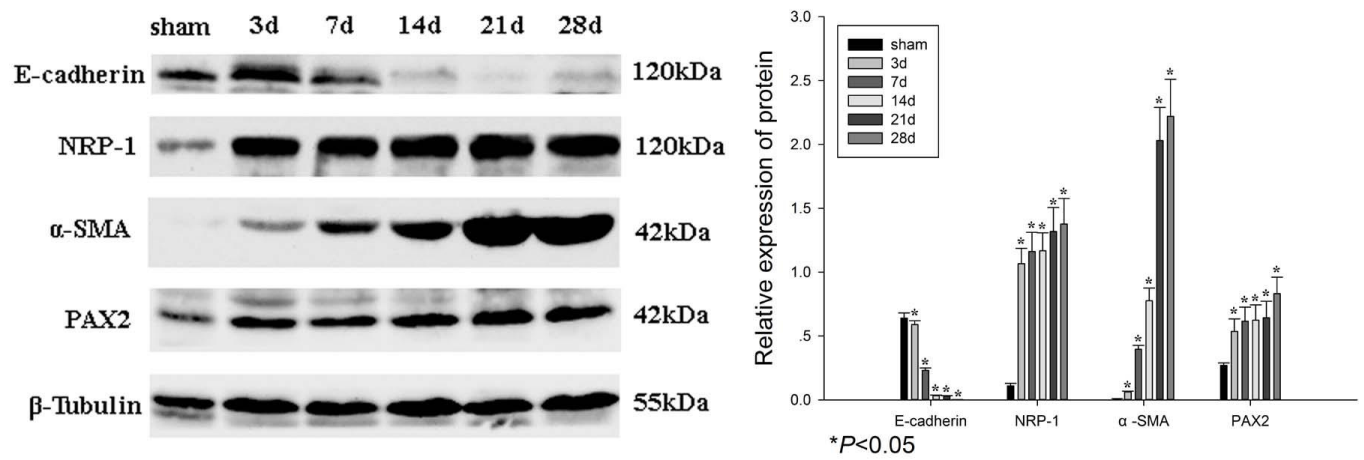

Figure 7. Protein expression of E-cadherin, NRP-1, ham group and the UUO group at various time points detected by immunohistochemistry (400ncrease of $(* \mathrm{P}<0.05$, UUO group vs sham group) 


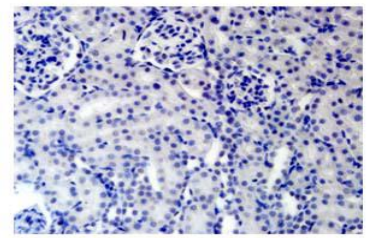

A

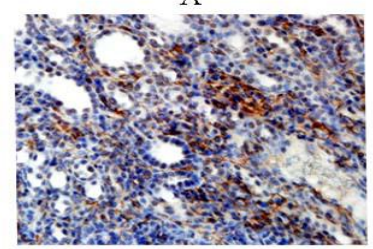

D

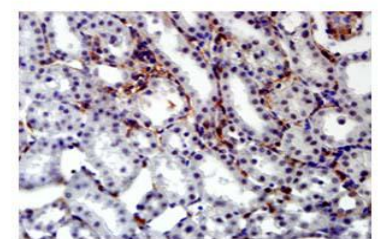

$\mathrm{B}$

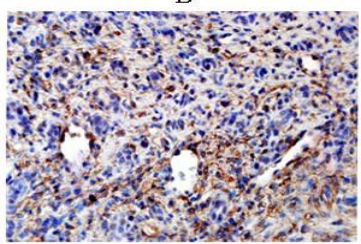

$\mathrm{E}$

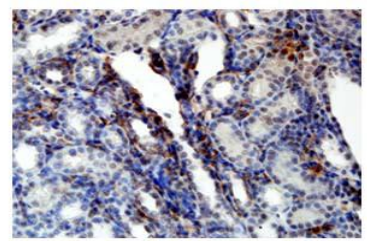

C

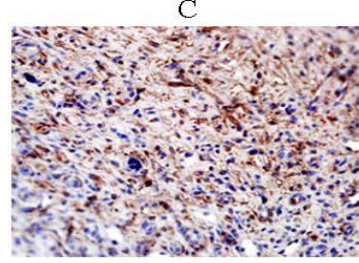

F

Figure 8. Protein expression of E-cadherin, NRP-1, ham group and thegroup at various time points detected by immunohistochemistry $(400 \times)$. A: sham group; B: 3d group; C: 7d group; D; 14d group; E: 21d group; F: 28d group.
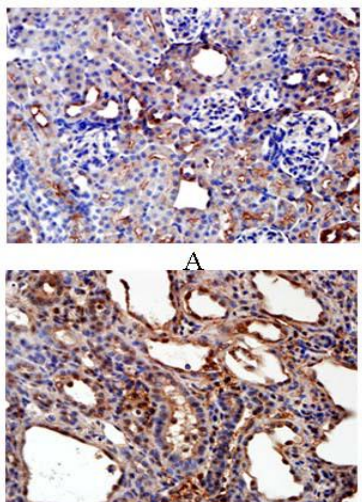

$\mathrm{D}$

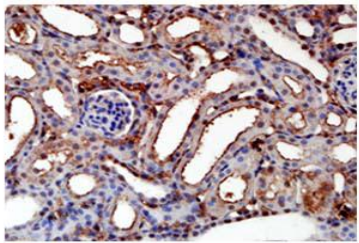

B

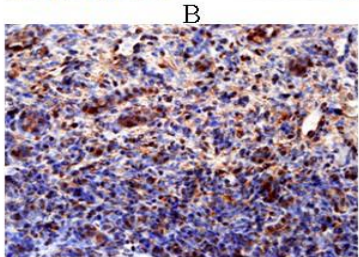

$\mathrm{E}$
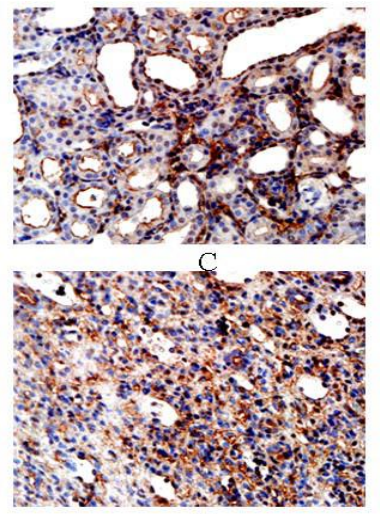

F

Figure 9. Protein expression of NRP-1 in the sham group and the UUO group at various time points detected by immunohistochemistry $(400 \times)$. A: sham group; B: 3d group; C: 7d group; D; 14d group; E: 21d group; F: 28d group.

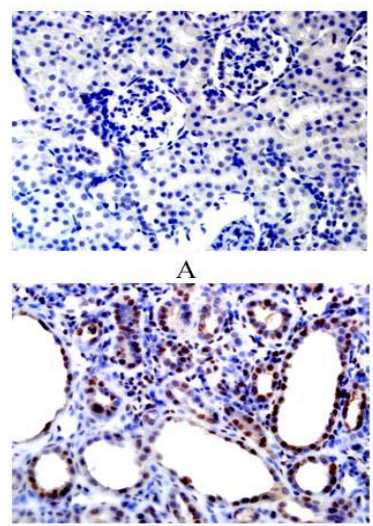

$\mathrm{D}$

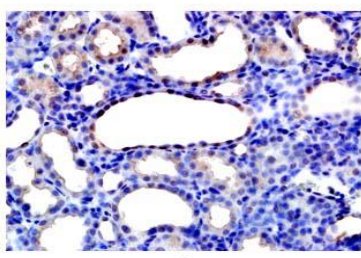

B

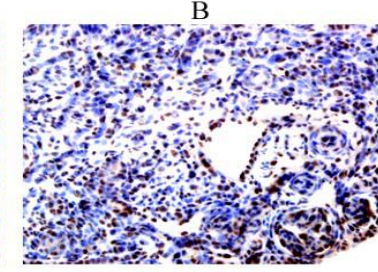

$\mathrm{E}$
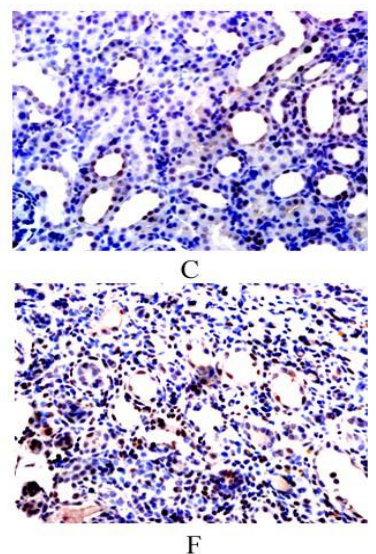

Figure 10. Protein expression of PAX2 in the sham group and the UUO group at various time points detected by immunohistochemistry $(400 \times)$. A: sham group; B: 3d group; C: 7d group; D; 14d group; E: 21d group; F: 28d group. 


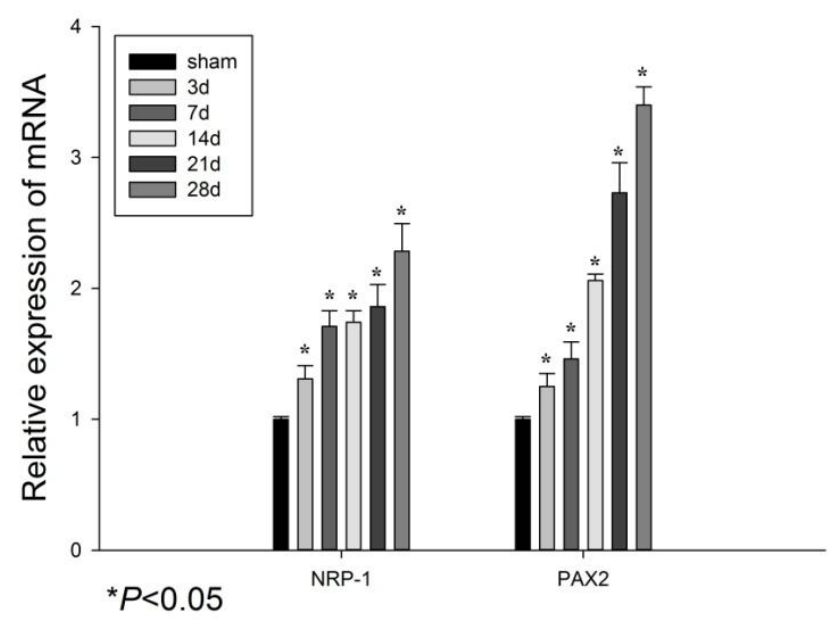

Figure 11. Messenger RNA expression of NRP-1 and PAX2 in the sham group and the UUO group at various time points detected by Real-time $\mathrm{qPCR}(* \mathrm{P}<0.05$, UUO group vs sham group).

\section{Discussion}

The EMT of renal tubular epithelial cells is a key event underlying renal fibrosis ${ }^{[12]}$. During EMT process, the epithelial cells lose their polarity and surface markers due to dedifferentiation, and induce the expression of mesenchymal markers, and gradually causing the formation of myofibroblasts. In a preliminary study, we found that PAX2 induces EMT in normal renal tubular epithelial cells in vitro ${ }^{[8]}$. By rat cDNA microarray screening, we selected a candidate target gene, NRP-1, which was differentially expressed in response to PAX2 over-expression. NRP-1, initially identified as a nerve cell surface protein ${ }^{[13]}$, is a transmembrane glycoprotein with a relative molecular weight of 1.3 wei5 and contains large extracellular domains to interact with semaphorin 3A, VEGF family and ligands (e.g., hepatocyte growth factor, platelet derived growth factor of 1.3 of 1.3NRP-1, whpithelial cells thelial cells ces EMT in ${ }^{[14,15]}$.

The NRP1-transgenic and NRP1-knockout mouse and zebrafish models confirmed that NRP-1 plays an important role in the development of embryonic nerves and cardiovascular system ${ }^{[16-18]}$. Besides, it was reported that NRP1 might mediate the primary immune response and the regeneration and repair of tissues ${ }^{[19]}$. NRP1, which is highly expressed in different tumor cell lines and human tumors, participates in the growth and vascularization of tumors ${ }^{[20-21]}$. These findings show that NRP1 is a multi-functional common receptor for the development of embryonic nerves and cardiovascular system, and may be involved in other pathophysiological processes.

The role of NRP1 in kidneys has not been studied extensively as yet. In a study, Villegas et al. found that NRP1 was markedly overexpressed in embryonic kidneys than in mature kidneys, and it was mainly expressed in the developing renal glomeruli and normal human renal tubular epithelial cells and collecting ducts ${ }^{[22]}$. A study by Karihaloo et al. showed that VEGFs might induce the morphogenesis of renal epithelial cells and tubulogenesis via an NRP1-dependent pathway ${ }^{[23]}$. However, the expression of NRP1 in renal tubular epithelial cells and its role in the occurrence of RIF have not been reported yet.

Studies have suggested that overexpressed NRP-1 can induce EMT in oral squamous cell carcinoma cells and human gastric cancer cells ${ }^{[10,24]}$. Three signaling pathways are involved in EMT induced by NRP-1: TGF-induce EMT in oral scMet. During EMT induction, there are interactions between TGF-ere induced byWnt, Ras, Hedgehog, or Notch pathway ${ }^{[15]}$. The role of NRP-1 in the EMT of renal tubular epithelial cells has not been studied as yet. This study initially demonstrated that PAX2 increased expression of NRP-1 mRNA and protein when it induced EMT in rat renal tubular epithelial cells, which validates the microarray data and suggests that NRP-1, as a downstream gene of PAX2, may play an important role in EMT induced by PAX2. Our study provides indirect evidence that NRP-1 promotes the EMT of renal tubular epithelial cells.

NRP-1 promotes hepatic cirrhosis in mice/rats and human beings via activating PDGF/TGF-csignaling pathways in hepatic stellate cells ${ }^{[1]}$, but there are no study reports about its role in renal fibrosis. By establishing a UUO rat model of renal fibrosis, in this study, we observed enhanced expression of NRP-1 in obstructed kidneys and that the expression of NRP-1 was coincident with the expression of PAX2 and renal fibrosis. This provides evidence that NRP-1 may play an important role in the process of RIF and may be a potential target for the clinical treatment of RIF.

In conclusion, our experiment for the first time provides in vitro and in vivo evidence of the possible involvement of NRP-1 in EMT induced by PAX2 in renal tubular epithelial cells, and the possible important role of NRP-1 in the process of RIF. Thus, our study may shed light on the pathogenesis of renal fibrosis with respect to the involvement of NRP-1, and aid in the development of anti-fibrotic therapeutic strategies. However, this study has certain limitations: for example, overexpression of exogenous NRP-1 or blockade of endogenous NRP-1 expression in renal tubular epithelial cells were not investigated. Additionally, the mechanism by which NRP-1 plays a role in RIF warrants further in-depth 
studies.

\section{Conclusion}

In conclusion, our experiment for the first time provides in vitro and in vivo evidence of the possible involvement of NRP-1 in EMT induced by PAX2 in renal tubular epithelial cells, and the possible important role of NRP-1 in the process of RIF. Thus, our study may shed light on the pathogenesis of renal fibrosis with respect to the involvement of NRP-1, and aid in the development of anti-fibrotic therapeutic strategies. However, this study has certain limitations: for example, overexpression of exogenous NRP-1 or blockade of endogenous NRP-1 expression in renal tubular epithelial cells were not investigated. Additionally, the mechanism by which NRP-1 plays a role in RIF warrants further in-depth studies.

\section{Acknowledgements (as necessary)}

This work was partially supported by grants from the Natural Science Foundation of Liaoning Province, China (2013225086, 2013021099, 2015020492), Science and Technology Planning Project of Shenyang City, China (F13-2219-59) and Excellent Doctoral Project Fund of Shengjing Hospital of China Medical University (MC96).

\section{Declaration of conflicting interests}

The author(s) declared no potential conflicts of interest with respect to the research, authorship, and/or publication of this article.

\section{Ethical statement}

With the approval by the Ethics Committee of Shengjing Hospital of China Medical University (2016PS006K).

\section{References}

1. Meran S, Steadman R. Fibroblasts and myofibroblasts in renal fibrosis. Int J ExpPathol 2011, 92(3): 158-167. doi: 10.1111/j.1365-2613.2011.00764.x.

2. Liu Y. Epithelial to mesenchymal transition in renal fibrogenesis: Pathologic significance, molecular mechanism, and therapeutic intervention. J Am Soc Nephrol 2004, 15(1): 1-12. doi: 10.1097/01.asn.0000106015.29070.e7.

3. Bouchard M, Souabni A, Mandler M, et al. Nephric lineage specification by Pax2 and Pax8. Genes Dve 2002, 16(22): 2958-2970. doi: 10.1101/gad.240102.

4. Grote D, Souabni A, Busslinger M, et al. Pax2/8-regulated Gata3 expression is necessary for morphogenesis and guidance of the nephric duct in the developing kidney. Development 2005, 133(1): 53-61. doi: 10.1242/dev.02184.

5. Murer L, Caridi G, Della Vella M, et al. Expression of nuclear transcription factor PAX2 in renal biopsies of juvenile nephronophthisis. Nephrol 2002, 91(4): 588-593. doi: 10.1159/000065017.

6. Li L, Wu Y, Zhang W. PAX2 re-expression in renal tubular epithelial cells and correlation with renal interstitial fibrosis of rats with obstructive nephropathy. Ren Fail 2010, 32(5): 603-611. doi: 10.3109/08860221003778049.

7. Li L, Wu Y, Wang C, et al. Inhibition of PAX2 gene expression by siRNA (polyethylenimine) in experimental model of obstructive nephropathy. Ren Fail 2012, 34(10): 1288-1296. doi: 10.3109/0886022X.2012.723662.

8. Li L, Wu Y, Yang Y. Paired box 2 induces epithelial-mesenchymal transition in normal renal tubular epithelial cells of rats. Mol Med Rep 2013, 7(5): 1549-1554. doi: 10.3892/mmr.2013.1365.

9. Wild JR, Staton CA, Chapple K, et al. Neuropilins: Expression and roles in the epithelium. Int J ExpPathol 2012, 93(2): 81-103. doi: 10.1111/j.1365-2613.2012.00810.x.

10. Chu W, Song X, Yang X, et al. Neuropilin-1 promotes epithelial-to-mesenchymal transition by stimulating nuclear factor-kappa B and is associated with poor prognosis in human oral squamous cell carcinoma. PLoSone 2014, 9(7): e101931. doi: 10.1371/journal.pone.0101931.

11. Cao S, Yaqoob U, Das A, et al. Neuropilin-1 promotes cirrhosis of the rodent and human liver by enhancing PDGF TGF-beta signaling in hepatic stellate cells. J Clin Invest 2010, 120(7): 2379-2394. doi: 10.1172/JCI41203.

12. Rastaldi MP. Epithelial-mesenchymal transition and its implication for the development of renal tubulointerstitial fibrosis. J Nephrol 2006, 19(4): 407-412. doi: 10.1152/ajprenal.90539.2008.

13. Takagi S, Tsuji T, Amagai T, et al. Specific cell surface labels in the visual centers of Xenopuslaevis tadpole identified using monoclonal antibodies. Dev Biol 1987, 122(1): 90-100. doi: 10.1016/0012-1606(87)90335-6.

14. Schramek H, Sarközi R, Lauterberg C, et al. Neuropilin-1 and neuropilin-2 are differentially expressed in human proteinuric nephropathies and cytokine-stimulated proximal tubular cells. Lab Invest 2009, 89(11):1304-1316. doi: 10.1038/labinvest.2009.96.

15. Prud'homme GJ, Glinka Y. Neuropilins are multifunctional coreceptorsinvolved in tumor initiation, growth, metastasis and immunity. Oncotarget 2012,3(9): 921-939. doi: 10.18632/oncotarget.626.

16. Gu C, Rodriguez ER, Reimert DV, et al. Neuropilin-1 conveyssemaphorin and VEGF signaling during neural and 
cardiovasculardevelopment. Dev Cell 2003, 5(1): 45-57. doi: 10.1016/s1534-5807(03)00169-2.

17. Kitsukawa T, Shimizu M, Sanbo M, et al. Neuropilin-semaphorin III/Dmediatedchemorepulsive signals play a crucial role in peripheral nerveprojection in mice. Neuron 1997, 19(5): 995-1005. doi: 10.1016/s0896-6273(00)80392-x.

18. Lee P, Goishi K, Davidson AJ, et al. Neuropilin-1 is required for vasculardevelopment and is a mediator of VEGFdependent angiogenesis inzebrafish. Proc Natl AcadSci USA 2002, 99(16): 10470-10475. doi: $10.1073 /$ pnas.162366299.

19. Staton CA, Kumar I, Reed MWR, et al. Neuropilins in physiological andpathological angiogenesis. J Pathol 2007, 212(3): 237-248. doi: 10.1002/path.2182.

20. Pan Q, Chanthery Y, Liang WC, et al. Blocking neuropilin-1 function hasan additive effect with anti-VEGF to inhibit tumor growth. Cancer Cell 2007, 11(1): 53-67. doi: 10.1016/j.ccr.2006.10.018.

21. Guttmann-Raviv N, Kessler O, Shraga-Heled N, et al. The neuropilins and their role in tumorigenesis and tumor progression. Cancer Lett 2006, 231(1): 1-11. doi 10.1016/j.canlet.2004.12.047.

22. Villegas G, Tufro A. Ontogeny of semaphorins $3 \mathrm{~A}$ and $3 \mathrm{~F}$ and their receptors neuropilin 1 and 2 in the kidney. Gene Expr 2002, Patterns 2, 151-155. doi: 10.1016/s0925-4773(02)00305-2.

23. Karihaloo A, Karumanchi SA, et al. Vascular endothelial growth factor induces branching morphogenesis/tubulogenesis in renal epithelial cells in a neuropillin-dependent fashion. Mol Cell Biol 2005, 25(17): 7441-7448. doi: 10.1128/MCB.25.17.7441-7448.2005.

24. Peng Y, Liu YM, Li LC, et al. MicroRNA-338 Inhibits Growth, Invasion and Metastasis of Gastric Cancer by Targeting NRP1 Expression. PLoS One 2014, 9(4): e94422. doi: 10.1371/journal.pone.0094422 